\title{
Road traffic mortality attributable to alcohol in Russia
}

\author{
Razvodovsky YE* \\ Grodno State Medical University, 80 Gorky Street, Grodno 230009, Belarus
}

\begin{abstract}
Background: The road accident mortality rates in Russia are the highest in Europe. The growing evidence suggests that drunk driving is the leading cause of fatal road accidents in Russia.

Objectives: The aim of the present study was to evaluate the effect of changes in aggregate-level alcohol consumption on fatal road traffic accidents in Russia between 1970 and 2015.

Methods: Age-standardized sex-specific male and female traffic accidents mortality data for the period 1970-2015 and data on alcohol consumption per capita were analyzed by means ARIMA (autoregressive integrated moving average) time series analysis.

Results: Alcohol consumption was significantly associated with both male and female traffic accidents mortality rates: a 1 liter increase in overall alcohol consumption would result in a $3.5 \%$ increase in the male accident mortality rate and in $2.1 \%$ increase in the female mortality rate. The results of the analysis suggest that $38.3 \%$ of all male accident deaths and $25.2 \%$ female deaths in Russia could be attributed to alcohol.
\end{abstract}

Conclusions: This is the first time-series analysis of overall level of alcohol consumption and road traffic mortality rates in Russia, which has shown that population drinking, is the strong predictor of road traffic fatalities at the aggregate level.

\section{Introduction}

Road traffic accidents are among the leading cause of mortality and a major public health issue in the many countries of the world $[1,2]$. According to WHO prognosis, road traffic mortality will become the fifth leading cause of deaths in the world, resulting in an approximately 2.4 million deaths annually [2]. Driving while under the influence of alcohol (drunk driving) has been recognized as one of the most important risk factors of fatal road traffic crashes [3,4]. There is causal, dose-response relationship between alcohol drinking and road traffic accidents, with risk of crashes increasing non-liner with increasing alcohol consumption [2]. The fraction of road traffic mortality attributable to alcohol varies from country to country. According to the estimates up to 48 percent of road traffic deaths in the EU countries are attributable to alcohol [3].

The road accident mortality rates in Russia are the highest in Europe $[5,6]$. The growing evidence suggests that drunk driving is the leading cause of fatal road accidents in Russia [3,7]. During the last decade, almost 40000 Russians have died in road accidents caused by drunk drivers [6]. A large retrospective study reported doseresponse relationship between binge drinking and mortality from road accidents: drinking of three or more bottles of vodka per week was strongly associated with deaths from road accidents both among men and women [8]. The most recent individual-level estimates suggest that $46.1 \%$ of all deaths from road accidents $(47.7 \%$ and $41.0 \%$ of deaths among men and women respectively) were attributable to alcohol consumption [9].

Despite the growing evidence that binge drinking is the major determinant of high road traffic mortality rate in Russia, there has been no prior time-series analysis of alcohol consumption and road traffic fatalities in this country. In relation to this, the aim of the present study was to evaluate the effect of changes in aggregate-level alcohol consumption on fatal road traffic accidents in Russia between 1970 and 2015.

\section{Material and methods}

\section{Data}

The data on sex-specific road traffic fatality rates per 1000000 of the population were taken from the Russian vital statistics registration system. Estimation of alcohol consumption per capita was based on the classical indicator of alcohol-related problem (alcohol poisoning mortality and alcohol psychoses incidence rates) which was adjusted for the effect of recorded alcohol consumption employing ARIMA (autoregressive integrated moving average) model [10].

\section{Statistical analysis}

We used autoregressive integrated moving average techniques (ARIMA), that has been suggested by Box \& Jenkins [11], to estimate the relationships between the annual per capita alcohol consumption and traffic accidents mortality time series in Russia. Since the bevariate correlations between two raw time-series are usually spurious due to common trends [12], these trends are removed by means of a differencing procedure. This procedure reduces substantially the risk

Correspondence to: Y. E. Razvodovsky, Grodno State Medical University, 80 Gorky Street, Grodno 230009, Belarus, E-mail: razvodovsky@tut.by

Key words: road traffic accidents, mortality, alcohol consumption, ARIMA time series analysis, Russia, 1970-2015

Received: October 24, 2016; Accepted: November 23, 2016; Published: November 26, 2016 
of spurious correlations. The process of removing the confounding variations is referred to as "prewhitening".

The effect of alcohol consumption on fatal road traffic accidents rates will be expressed in terms of alcohol-attributable fraction (AAF), which is interpreted as the proportion of accident deaths that is attributable to alcohol. AAF can be calculated from the estimates obtained in ARIMA models according to formula: AAF $=1-\exp (-\mathrm{bX})$, where $\mathrm{X}$ is alcohol consumption per capita in the study period and $\mathrm{b}$ is the estimated effect parameter [12]. To examine the relation between changes in the alcohol consumption and traffic accident mortality across the study period a time-series analysis was performed using the statistical package "Statistica".

\section{Results}

Across the whole period the male traffic accidents mortality rates was 3.8 times higher than the female rates ( 442.3 vs. 115.2 per 1000.000 ) with a rates ratio of 5.0 in 1970 decreasing to 3.4 by the 2015 . This may reflect a relatively new phenomenon in Russia: a growing number of women drivers [6]. The trends in the sex-specific traffic accidents mortality rates are displayed in Figures 1-2. As can be seen, the pattern of accidents mortality for men and women was uniform. For both sexes the time series accidents mortality rates fluctuated greatly over the period: increased from 1970 to 1977 , decreased markedly between
1981-1984 (by $13.1 \%$ for men and 14,5\% for women), dropped sharply between $1984-1986$ (by $27.0 \%$ for men and $7.8 \%$ for women), than jumped dramatically between 1987 and 1991 (by 64.2\% for men and $53.4 \%$ for women). From 1993-1997 there was a fall in the rates before they again rising between 1997 and 2003, and than started to decrease. There was also a spike in mortality in 2012 .

The graphical evidence suggests that the trends in both alcohol consumption per capita and traffic accidents mortality for males and females seem to follow each other across the time-series (Figures 1-2). There were S-shape and linear trends in the time series data across the study period. These systematic variation was well accounted for by the application of first-order differencing. All of the final univariate models meet the diagnostic criterion (i.e., none of the autocorrelation functions are significant at the 0.05 level). After prewhitening the cross-correlations between alcohol consumption and traffic accidents mortality time series were inspected. There was a statistically significant cross-correlation between per capita alcohol consumption and traffic accidents mortality for males and females at lag zero.

The specification of the bivariate ARIMA model and outcome of the analyses are presented in Table 1. According to the results, alcohol consumption is a statistically significant associated with both male and female traffic accidents mortality rates, implying that a 1-litre increase

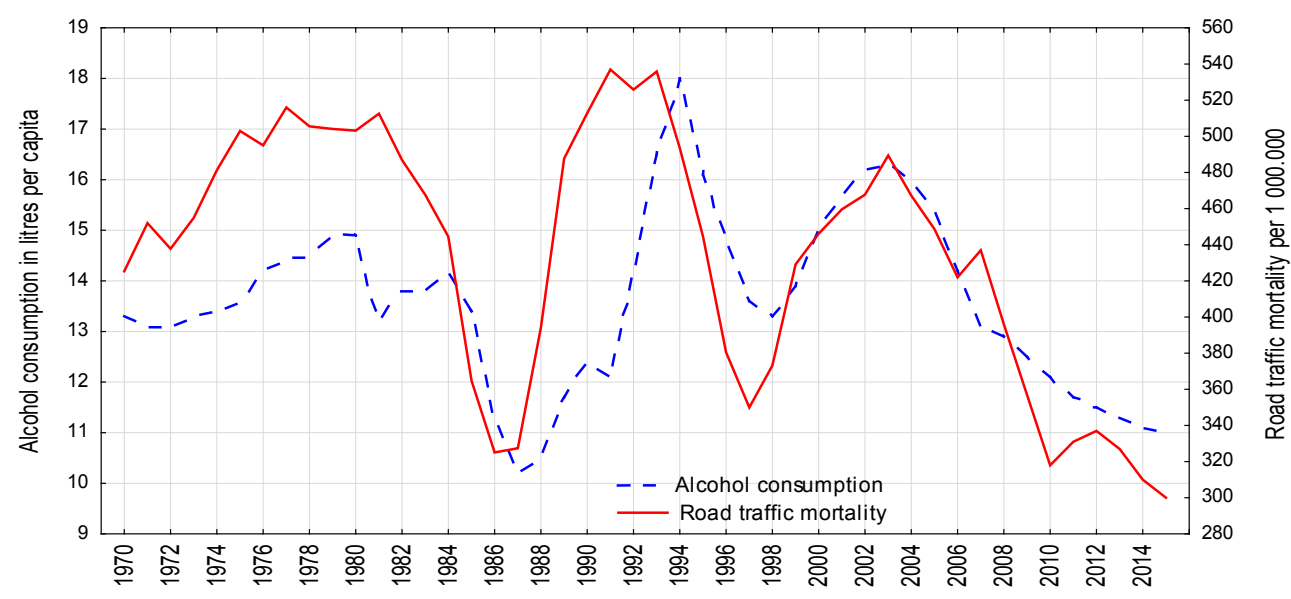

Figure 1. Trends in alcohol consumption per capita and male traffic accidents mortality in Russia 1970 and 2015.

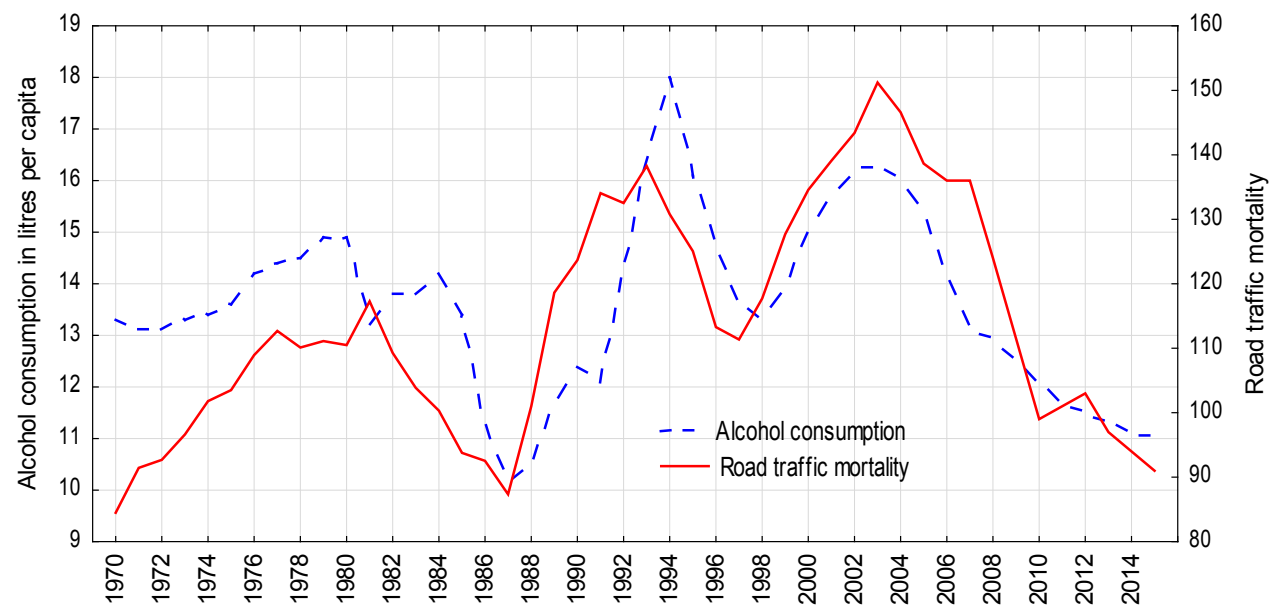

Figure 2. Trends in alcohol consumption per capita and female traffic accidents mortality in Russia 1970 and 2015. 
Table 1. Estimated effects (bivariate ARIMA model) of overall alcohol consumption on traffic accident mortality rates and alcohol-attributable deaths to all accident deaths by gender.

\begin{tabular}{|l|c|c|c|c|}
\hline Parameter & Model & Estim. & P & AAF \\
\hline Mortality males & $0.1 .1^{*}$ & 0.035 & 0.008 & 38.3 \\
\hline Mortality females & 0.1 .1 & 0.021 & 0.039 & 25.2 \\
\hline
\end{tabular}

*The general form of non-seasonal ARIMA model is $(\mathrm{p}, \mathrm{d}, \mathrm{q})$, where $\mathrm{p}$ - the order of the autoregressive parameter, $\mathrm{d}$ - the order of differencing, and $\mathrm{q}-$ the order of the moving average parameter. $\mathrm{Q}$ test for residuals are satisfactory in all models.

in per capita alcohol consumption is associated with an increase in male mortality of $3.5 \%$ and female mortality of $2.1 \%$. Table 1 also shows the relative proportion of alcohol-attributable deaths to all traffic accidents deaths by gender. The results of the analysis suggest that $38.3 \%$ of all male traffic accidents deaths and $25.2 \%$ female deaths in Russia could be attributed to alcohol.

\section{Discussion}

The results of the time-series analysis suggest a positive and statistically significant relationship between traffic accidents mortality rates and population drinking in Russia. Estimation the proportion of alcohol-attributable fraction indicates that $38.3 \%$ of male deaths and $25.2 \%$ of female deaths in Russia are attributable to alcohol. These aggregate-level findings are comparable to previous individual-level estimates for males (37.0\%), but exceed substantially estimates for females (9.2\%) [3]. At the same time, these results are in contrast to those reported by Shield \& Rehm, who found that $41.0 \%$ of all deaths from traffic accidents among women attributable to hazardous drinking [9]. In an international comparison, Russia stood out with an alcohol effect that was larger than what had previously been estimated for other countries [2].

The empirical evidence, presented in this study, suggests that traffic accidents mortality rates can be controlled through alcohol availability measures. Indeed, the substantial decline in the traffic accidents mortality rates in the mid-1980s corresponds with the anti-alcohol campaigns, which Gorbachev launched in may 1985 [4,13,14]. This campaign consisted of several measures designed to limit the availability and affordability of alcohol by raising the price of vodka, and placing the restriction on alcohol sale by amount and time of day $[13,15]$. As a result, official figures indicated a marked reduction in alcohol consumption. There were also recorded decline of similar magnitude in road traffic deaths. The deaths rate climbs rapidly between 1987 and 1991, and this increase, most probably, was associated with the end of anti-alcohol campaign in 1987.

It should be emphasized that there has been greater proportional declining in the numbers of male deaths in fatal traffic accidents during anti-alcohol campaigns: between 1984 and 1986 the number of male deaths declined by $27.0 \%$, whereas the number of female deaths only declined by $7.8 \%$. This fact suggests that males are more likely than females to be involved in alcohol-related fatal crashes and that man are more affected by the alcohol availability control measures.

Strikingly, but the road traffic mortality rates after reaching its pick in 1991, started to decrease, despite the growing level of alcohol consumption. Given the evidence that the number of road traffic deaths is inversely related to economic performance [16], it would be logical to assume that the downward trend in road traffic mortality rates in the mid of 1990s is related to the crisis that hit Russian economy after the collapse of Soviet Union in 1991. The decrease in traffic-related fatalities during the economic crisis in the 1990s was also recorded in other former Soviet Republics [17]. A similar pattern of road traffic mortality rates during the economic crisis was observed in other regions [16].

In Russia, traffic accidents mortality rates has declined substantially since 2003, with the highest rates of decline in 2007 and 2010. There is suggestive evidence that the new alcohol policies, introduced by Russian government in 2006, played the important role in this mortality decline $[18,19]$. The new alcohol policies measures include tightened regulation of both production and sale of alcohol [20]. These measures have overlapped with efforts to reduce road traffic mortality through stricter drunk driving laws. In 2005 the government approved a Federal Target Program for Ensuring Road Traffic Safety. Further, in 2007 higher fines for drunk driving were introduced [6.

In his analysis of monthly data Pridemore and coauthors [21] showed that the implementation of new alcohol policies was responsible for an $11 \%$ reduction in male deaths due to transport accidents or saving more than 2400 males lives annually. However, the implementation of the suite of alcohol control policies had no impact on female deaths due to transport accidents. This research evidence suggests that recent Russian government's attempt to reduce the alcohol-related mortality rates have been at least partially successful. It might be, however, that both reduction in alcohol consumption and road traffic mortality decline in Russia resulted from variety of other factors, including falling unemployment, rising incomes and improved health care [22].

In conclusion, this is the first time-series analysis of overall level of alcohol consumption and traffic accidents mortality rates in Russia, which has shown that population drinking, is the strong predictor of road traffic fatalities at the aggregate level. This study present convincing evidence that a restrictive alcohol policy can be considered as an effective measure of road traffic mortality prevention. In relation to this, Russian authorities should pay more attention to the problem of drunk driving. A greater emphasis on effective alcohol-control policies needed to limit the danger of drinking while under the influence of alcohol.

\section{References}

1. Road Safety Performance - National Peer Review: Russia Federation. OECD/ITF. 2011

2. World Health Organization. Global status report on road safety: time for action Geneva: World Health Organization; 2009.

3. Drink Driving: Towards Zero Tolerance European transport safety council Brussels 2012

4. Razvodovsky YE (2016) The Effect of Gorbachev's Anti-alcohol Campaign on Road Traffic Accidents Mortality in Belarus. J Alcohol Drug Depend 4: 241.

5. Kandrychyn SV, Razvodovsky YE (2015) The spatial regularities of violent mortality in European Russia and Belarus: ethnic and historical perspective. J Psychiatry 18: 305.

6. Petrova E (2013) Road accidents in Russia: statistical and geographical analysis. Scientific Annals of "Alexandru Ion Cuza University of Iasi" 59(2): 11-113.

7. Razvodovsky YE (2010) Beverage specific alcohol sale and mortality in Russia Alcoholism 46(2): 63-75

8. Zaridze D, Maximovitch D, Lazarev A, Igitov V, Boroda A, et al. (2008) Alcohol poisoning is a main determinant of recent mortality trends in Russia: evidence from a detailed analysis of mortality statistics and autopsies. Int J Epidemiol 38:143-153. [Crossref]

9. Shield KD, Rehm J (2015) Russia-specific relative risks and their effects on the estimated alcohol attributable burden of disease. BMC Public Health 15: 482. [Crossref]

10. Razvodovsky YE (2013) Estimation of the level of alcohol consumption in Russia ICAP Periodic Review Drinking and Culture 8: 6-10. 
11. Box GEP, Jenkins GM (1976) Time Series Analysis: forecasting and control. London. Holden-Day Inc

12. Norström T (1989) The use of aggregate data in alcohol epidemiology. Br J Addict 84 969-977. [Crossref]

13. Nemtsov AV (2011) A contemporary history of alcohol in Russia. Stockholm. Sodertorns hogskola.

14. Nemtsov AV, Razvodovsky YE (2008) Alcohol situation in Russia, 1980-2005. Social and Clinical Psychiatry 2: 52-60.

15. Stickley A, Leinsalu M, Andreev E, Razvodovsky Y, Vågerö D, et al. (2007) Alcohol poisoning in Russia and the countries in the European part of the former Soviet Union, 1970 2002. Eur J Public Health 17: 444-449. [Crossref]

16. Why does road safety improve when economic times are hard? International Traffic Safety Data and Analysis Group. Research Report 2015.
17. Razvodovsky YE (2003) Association between distilled spirits consumption and violent mortality rate. Drugs: Education, Prevention and Policy 10: 235-250.

18. Razvodovsky YE, Nemtsov AV (2016) Alcohol-related component of the mortality decline in Russia after 2003. The Questions of Narcology 3: 63-70.

19. Razvodovsky YE (2014) Was the mortality decline in Russia attributable to alcohol control policy? Journal of Sociolomics 3: 1-2.

20. Nemtsov AV, Razvodovsky YE (2016) Russian Alcohol Policy in False Mirror. Alcohol Alcohol 51: 626-627. [Crossref]

21. Pridemore WA, Chamlin MB, Kaylen MT, Andreev E (2013) The impact of a national alcohol policy on deaths due to transport accidents in Russia. Addiction 108: 2112 2118. [Crossref]

22. He H, Paichadze N, Hyder AA, Bishai D (2015) Economic development and road traffic fatalities in Russia: analysis of federal regions 2004-2011. Inj Epidemiol 2: 19. [Crossref]

Copyright: (C2016 Razvodovsky YE. This is an open-access article distributed under the terms of the Creative Commons Attribution License, which permits unrestricted use, distribution, and reproduction in any medium, provided the original author and source are credited. 